\title{
Assessment of Neutrophil Lymphocyte Ratio in Systemic Sclerosis Patients in Tanta University Hospital: A Promising Marker in Predicting Disease Severity
}

\author{
Noha E. Esheba, Abeer Shahba \\ Department of Internal Medicine, Tanta University; Egypt
}

\begin{abstract}
Introduction: Systemic sclerosis (SSc) is a multisystem autoimmune disease, with complex pathogenesis resulting in obliterative vasculopathy, tissue injury, fibrosis, remodeling and atrophy. The neutrophil lymphocyte ratio (NLR) was developed to provide easily measurable and readily available parameter reflecting the intensity of stress and systemic inflammation in critically ill patients following shock, multiple traumas, major surgery, or sepsis. Aim of the work: to evaluate NLR levels in patients with SSc and explore their clinical significance and their association with different organ manifestations. Results: There was a significant increase in NLR in SSc patients who had cardiovascular or cardiorespiratory affection. Also NLR showed a significant positive correlation with both CRP and ESR, while it showed a significant negative correlation with serum albumin. Conclusion: NLR may serve as a promising marker for cardiorespiratory involvement in SSc patients. [Egypt J Rheumatology \& Clinical Immunology, 2016; 4(1): 43-47]
\end{abstract}

Key Words: Systemic sclerosis, Neutrophil lymphocyte ratio, SSc, NLR.

\section{INTRODUCTION}

Systemic sclerosis (SSc) is a systemic autoimmune disease characterized by generalized microvascular lesions and obliterative arterial vasculopathy ${ }^{1}$.

There are variable organ affection in patients with SSc, including left diastolic heart dysfunction, vascular lesion of the kidney (scleroderma renal crisis) and peripheral arterial disease. Lung and skin fibrosis may also coincide with impaired microcirculation ${ }^{2-6}$.

Markers of inflammation such as increased CRP and ESR level, which may signify increased disease activity and unfavorable prognosis. Several studies showed that the level of ESR was significantly higher in patients with diffuse $\mathrm{SSc}$ than in those with localized $\mathrm{SSc}^{7,8}$

Neutrophil lymphocyte ratio (NLR) was considered to be a good marker for inflammation. NLR is increased in patients suffering from malignancies, such as colorectal, breast, and lung cancers; cardiovascular disease; and inflammatory disorders, like acute pancreatitis and ulcerative colitis 9 .

For our knowledge, the relationship between SSC and NLR has not been investigated yet.

The aim of this study was to investigate the association of Neutrophil lymphocyte ratio and the presence of different organ manifestations in patients with SSc.

\section{PATIENTS AND METHODS}

Twenty five patients ( 2 males and 23 females) with SSc were included in this study. The patient's data were collected from the rheumatology clinic files. The patient files were investigated retrospectively. Twenty five healthy subjects of matching age and sex were also included as a control group (2 males and 23 females). Patients older than 18 years and diagnosed to have SSC were included in the study, while those who had malignancy, active infection, diabetes mellitus, and liver failure were excluded from the study. All patients had diffuse SSc and fulfilled the criteria proposed by the American College of Rheumatology for $\mathrm{SSc}^{10}$. For each patient: pulmonary function tests, echocardiography were performed. Clinical characteristics included the presence of subcutaneous calcinosis, telangiectasia, skin hypo-/ hyperpigmentation and active skin ulcers. Skin thickness was scored according to the modified Rodnan skin thickness score (mRSS) by summing the skin thickness measurements as determined by palpation on a $0-3$ scale in 17 body areas (range $0-51)^{11,12}$. ESR, CRP, ANA, antiSCL 70, ACA and rheumatoid factor were also measured. Complete blood count was performed and NLR was found with a mathematical calculation of the ratio of neutrophils with lymphocytes. Internal organ manifestations were recorded according to a standard protocol as previously described ${ }^{3}$. SSc patients having a forced vital capacity (FVC) $<80 \%$ of the predicted value underwent high-resolution CT of the lungs for the 
assessment of interstitial lung disease. Left ventricular diastolic abnormality, pericarditis and conduction disturbances were also recorded based on ECG and echocardiography results. Severe micro-/ macrovascular involvement was considered in cases with active digital ulcers, amputation due to peripheral scleroderma-related vascular disease, cardiac conduction disturbance/ arrhythmia, left ventricular ejection fraction $<50 \%$, PAH or scleroderma renal crisis.

\section{Statistical Analysis}

Comparisons between two groups were performed using a two-tailed unpaired Student $t$ test. Data were presented as mean \pm standard deviation and range. Correlations were assessed by using the Pearson correlation test. $\mathrm{P}$ value less than 0.05 was considered statistically significant. All analyses were performed using SPSS statistical software (SPSS V.16, Inc., Chicago, IL).

\section{RESULTS}

The Demographic, laboratory and clinical data of SSc patients and controls were shown in Table (1). There was a significant increase in CRP and ESR in
SSC patients in comparison to controls $(\mathrm{P}<0.001$, $0.001)$ respectively.

The CBC findings in SSc patients in comparison to controls were shown in Table (2). When compared to controls, the $\mathrm{Hb}$ level was significantly decreased in SSc patients $(\mathrm{P}<0.001)$, while the leukocytes, neutrophils, lymphocytes and NLR were significantly increased $(\mathrm{P}<0.001$ for all).

Furthermore, there was a significant increase in NLR in SSc patients who had digital ulcers, pulmonary hypertension, left ventricle ejection fraction $<50 \%$, interstitial lung disease and vascular involvement ( patient had at least one of the following findings: $\mathrm{PAH}, \quad \mathrm{LVEF}<50 \%$, conduction disturbance/arrhythmia, active digital ulcers, scleroderma renal crisis, amputation due to severe peripheral arterial disease) in comparison to $\mathrm{SSc}$ patients who didn't have those lesions $(\mathrm{P}<0.001$ for all) as shown in Table (3).

As for some inflammatory markers, NLR showed a significant positive correlation with both CRP and ESR ( $\mathrm{P}<0.001$ for both), while it showed a significant negative correlation with serum albumin $(\mathrm{P}<0.01)$ as shown in table $(4)$

Table 1. Demographic, laboratory and clinical data of SSc patients and controls.

\begin{tabular}{|c|c|c|c|}
\hline Variable & SSc patients $N(25)$ & Controls N(25) & P-value \\
\hline Female N $(\%)$ & $23(92 \%)$ & $23(92 \%)$ & \\
\hline Age $($ mean $\pm \mathrm{SD})$ years & $41.05 \pm 13.46$ & $40 \pm 12.3$ & \\
\hline Disease duration (mean $\pm \mathrm{SD}$ ) years & $5.19 \pm 4.53$ & NA & \\
\hline $\mathrm{LVEF}<50 \% \mathrm{n}(\%)$ & $3(12 \%)$ & 0 & \\
\hline $\mathrm{PH}$ & $3(12 \%)$ & 0 & \\
\hline $\mathrm{FEV}_{1}<80 \% \mathrm{n}(\%)$ & $18(72 \%)$ & 0 & \\
\hline $\mathrm{FVC}<80 \% \mathrm{n}(\%)$ & $18(72 \%)$ & 0 & \\
\hline ILD by CT $\mathrm{n}(\%)$ & $15(60 \%)$ & 0 & \\
\hline SRC & $1(4 \%)$ & 0 & \\
\hline $\mathrm{ESR}($ mean $\pm \mathrm{SD}) \mathrm{mm} / \mathrm{h}$ & $43.05 \pm 32.24$ & $7.04 \pm 5.37$ & $<0.001$ \\
\hline $\mathrm{CRP}(\mathrm{mg} / \mathrm{dl})$ & $14.42 \pm 15.8$ & $0.75 \pm 2.05$ & $<0.001$ \\
\hline Albumin $(\mathrm{gm} / \mathrm{dl})$ & $3.8 \pm 1.03$ & $4.4 \pm 1.93$ & 0.008 \\
\hline Anti SCL 70 n (\%) & $10(40 \%)$ & 0 & \\
\hline ANA $n(\%)$ & $5(20 \%)$ & 0 & \\
\hline $\operatorname{ACA} n(\%)$ & 0 & 0 & \\
\hline RF n (\%) & $3(12 \%)$ & 0 & \\
\hline Digital ulcers/amputation $\mathrm{n}(\%)$ & $10(40 \%)$ & 0 & \\
\hline Pigment disorder & $13(52 \%)$ & 0 & \\
\hline
\end{tabular}

$\overline{\boldsymbol{A C A}}$ anticentromer antibody; $\boldsymbol{A N A}$ antinuclear antibody; $\boldsymbol{A n t i} \mathbf{S c l} \mathbf{7 0}$ anti sclerderma 70; $\boldsymbol{C R P} \mathrm{C}$ reactive protein; $\boldsymbol{E S R}$ erythrocyte sedimentation rate; $\boldsymbol{F} \boldsymbol{E} \boldsymbol{V}_{\boldsymbol{I}}$ forced expiratory volume 1; $\boldsymbol{F} \boldsymbol{V} \boldsymbol{C}$ forced vital capacity; $\boldsymbol{I} \boldsymbol{L D}$ interstitial lung disease; $\boldsymbol{L} \boldsymbol{V E F}$ left ventricular ejection fraction; $\boldsymbol{R} \boldsymbol{F}$ rheumatoid factor; $\boldsymbol{P H}$ pulmonary hypertension, $\boldsymbol{S} \boldsymbol{R} \boldsymbol{C}$ scleroderma renal crisis 
Table 2. CBC of SSc patients and the controls.

\begin{tabular}{lccc}
\hline \multicolumn{1}{c}{ Variable } & SSc patients & controls & P-value \\
\hline Hemoglobin $\mathrm{gm} / \mathrm{dl}$ & $11.08 \pm 1.69$ & $13.47 \pm 1.98$ & $<0.001$ \\
Platelet $\times 10^{3} / \mathrm{ml}$ & $225 \pm 107.82$ & $235 \pm 56.94$ & 0.09 \\
Leukocytes $\times 10^{3} / \mathrm{ml}$ & $7.94 \pm 4.09$ & $4.47 \pm 1.68$ & $<0.001$ \\
Neutrophils $\times 10^{3} / \mathrm{ml}$ & $5.15 \pm 2.89$ & $3.85 \pm 0.99$ & $<0.001$ \\
Lymphocytes $\times 10^{3} / \mathrm{ml}$ & $2.47 \pm 1.21$ & $1.94 \pm 0.42$ & $<0.001$ \\
Neutrophil Lymphocyte ratio & $2.34 \pm 1.04$ & $1.52 \pm 0.54$ & $<0.001$ \\
\hline
\end{tabular}

Table 3. Median NLR in SSc patients.

\begin{tabular}{lccc}
\hline \multirow{2}{*}{ Variable } & Present & Absent & \multirow{2}{*}{ P-value } \\
\cline { 2 - 3 } & \multicolumn{1}{c}{ NLR Median $\left(\mathbf{2 5}^{\text {th }} \mathbf{- 7 5}^{\text {th }} \mathbf{~ p e r c e n t i l e s ~}\right)$} & $<0.001$ \\
\hline Digital ulcers & $2.34 \pm 0.99$ & $2.01 \pm 0.82$ & 0.89 \\
Pigment disorder & $2.21 \pm 0.72$ & $2.20 \pm 0.72$ & $<0.001$ \\
PH & $3.9 \pm 0.56$ & $2.15 \pm 0.42$ & $<0.001$ \\
LVEF $<50 \%$ & $3.9 \pm 0.56$ & $2.15 \pm 0.42$ & $<0.001$ \\
ILD & $2.51 \pm 0.98$ & $2 \pm 0.91$ & 0.91 \\
dysphagia & $2.12 \pm 0.71$ & $2.11 \pm 0.69$ & $<0.001$ \\
Vascular ${ }^{1}$ & $2.31 \pm 0.51$ & $1.99 \pm 0.91$ & 0.9 \\
RF & $2.11 \pm 0.73$ & $2.1 \pm 0.74$ & \\
\hline
\end{tabular}

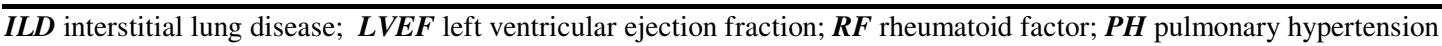

Table 4. Correlation of NLR with CRP and ESR.

\begin{tabular}{lcc}
\hline & Variable & \multicolumn{2}{c}{ Neutrophil lymphocyte ratio } \\
\cline { 2 - 3 } & Pearson $\mathbf{~}$ & P-value \\
\hline CRP & 0.45 & $<0.001$ \\
ESR & 0.34 & $<0.001$ \\
Albumin & -0.3 .01 & $<0.01$ \\
\hline
\end{tabular}

$\overline{C R P}$ C reactive protein; $\boldsymbol{E S R}$ erythrocyte sedimentation rate

\section{DISCUSSION}

SSc is a complicated autoimmune and connective disease, of which the pathogenesis and treatment have not been fully elucidated ${ }^{13}$.

Many studies have evaluated the clinical usefulness of Neutrophil lymphocyte ratio as a prognostic factor in many inflammatory disorders as: systemic lupus erythematosis, rheumatoid arthritis, ankylosing spondilitis and many others. After searching the literature, this is the first study showing the relation between NLR and SSc.

The relation between higher levels of NLR and systemic inflammation is not clear yet. Systemic inflammation affects vascular endothelial cells in a deleterious way, through decreasing the production of nitric oxide and prostacyclin, leading to decreased vasodilation and anti-thrombosis. Also, stimulating leukocytes causing increased adherence to the vascular endothelium $^{14}$.

Many cytokines, as interleukins (IL-1ra, IL-6, IL-7, IL-8, IL-12) and platelet derived growth factor (PDGF), take a leading role in the occurrence of inflammation and may have an impact on increased NLR ${ }^{15}$.

Neutrophil lymphocyte ratio is found to be related to numerous inflammatory disorders. Kaya et al. ${ }^{16}$ reported increased NLR to be a sign of severe atherosclerosis, and they suggested using NLR as a good marker for cardiac risk stratification. NLR has 
been found to be related with coronary atherosclerosis, and it is reported as an independent factor with CRP in predicting undesirable events in the hospital setting after myocardial infarction and unsuccessful attempt at primary percutaneous intervention ${ }^{17,18}$.

Furthermore, NLR has been shown be an marker for subclinical inflammation, and it was reported to be associated with prognosis in coronary artery disease and heart failure ${ }^{19,20}$.

Also in ulcerative colitis patients, studies reported that high levels of NLR were associated with disease activity, and they suggested the use of NLR as a marker for intestinal inflammation ${ }^{21,22}$. NLR was shown to be an independent predictor of mortality from ChildTurcotte-Pugh (CTP) and Model for End-Stage Liver Disease scores in patients with liver cirrhosis. NLR has a predictive role in overall and cancer-specific surveillance of many malignancies as malignant tumors of: stomach, lung, breast, and kidney ${ }^{23-25}$.

In patients with other inflammatory conditions, like acute pancreatitis and acute appendicitis, and systemic diseases, like hypertension, diabetes mellitus, and chronic renal failure, the levels of NLR were higher than normal ${ }^{26,27}$.

In patients with familial Mediterranean fever Ahsen et al. ${ }^{28}$ reported higher NLR levels in those patients when compared with the control group.

This study highlights the importance of NLR in reflection of the extent of inflammation and vascular involvement in SSC patients. When we investigated patients having at least one of the clinically significant macro- and/or microvascular organ manifestations in the form of (PAH, LVEF $<50 \%$, conduction disturbance/arrhythmia, active digital ulcers, scleroderma renal crisis or amputation due to severe peripheral arterial disease) we found a significantly higher mean NLR compared with cases without vascular symptoms (Table 3), indicating that NLR may be useful for the identification of patients with widespread vascular involvement. This indicates that neutrophils play an important role in the development of autoimmune responses in SSC patients.

In this study NLR was significantly higher in SSc patients with interstitial lung disease when compared with SSc without lung disease. It has been previously shown that lung disease coincides with microvascular lesions on nailfold capillaroscopy ${ }^{1,6}$.

NLR values also correlated with markers of inflammation (CRP, ESR), and (albumin). And it is known that activity of SSc involves ongoing vascular process and inflammation, therefore NLR may serve as a potential marker for disease activity and disease severity.

Further investigations are required to clarify the role of NLR as a good marker of disease activity in SSc. Furthermore, an increased NLR could be used clinically as a biomarker associated with decreasing LVEF and development of interstitial lung disease, indicating impairment of cardiovascular function and disease progression.

A limitation of the study is the small sample size of the patients group, and studies conducted on larger scale of patients are recommended to clarify the exact role of NLR in disease activity and progression. Another limitation was its retrospective design. Thus, a prospective study is needed to validate our results, and determine the possibility of using NLR as a prognostic tool or a guide for response to treatment, since Coskan et al 9 documented that Neutrophil lymphocyte ratio can be a valuable and reliable tool of disease activity in patients who have started antitumor necrosis factor (TNF) drugs for ankylosing spondylitis.

The strength of this study is the described results of increasing NLR with decreased cardiopulmonary function. Needless to say that estimation of NLR is easily measured, inexpensive and could contribute in monitoring cardiorespiratory involvement in patients with SSc.

\section{Conclusion}

There was significant increase in NLR in SSc patients compared with those of the controls. These findings provide evidence for the occurrence of a systemic inflammatory process in SSc, and suggest a potential role for NLR as a biomarker in evaluating patients with SSc.

NLR may serve as a promising marker for inflammation in SSc may be linked to lung involvement, and could contribute in monitoring cardiorespiratory involvement in patients with SSc, and may serve as a potential biomarker for this complication.

\section{Recommendation}

Further studies on larger number of patients and correlation with follow up and treatment received, are needed to elucidate the exact role of NLR in SSc patients.

\section{Conflict of interest}

The authors have none to declare.

\section{REFERENCES}

1. Kahaleh B. The microvascular endothelium in scleroderma. Rheumatology 2008;47(Suppl 5):v14 5.

2. Czirjak L, Kumanovics G, Varju C et al. Survival and causes of death in 366 Hungarian patients with systemic sclerosis. Ann Rheum Dis 2008; 67:59 63. 
3. Komocsi A, Pinter T, Faludi R et al. Overlap of coronary disease and pulmonary arterial hypertension in systemic sclerosis. Ann Rheum Dis 2010; 69:202 5.

4. Pinter T, Faludi R, Magyari B et al. Mechanism of coronary flow reserve reduction in systemic sclerosis: insight from intracoronary pressure wire studies. Rheumatology 2011; 50:781 8.

5. Picart C, Carpentier H, Brasseur S et al. Systemic sclerosis: blood rheometry and laser Doppler imaging of digital cutaneous microcirculation during local cold exposure. Clin Hemorheol Microcirc 1998;18:47 58.

6. Corrado A, Carpagnano GE, Gaudio A et al. Nailfold capillaroscopic findings in systemic sclerosis related lung fibrosis and in idiopathic lung fibrosis. Joint Bone Spine 2010;77:570 4

7. Nagy $Z$ and Czirjak L. Increased levels of amino terminal propeptide of type III procollagen are an unfavourable predictor of survival in systemic sclerosis. Clin Exp Rheumatol 2005; 23:165-72.

8. Farkas N, Szabo A, Lorand V et al. Clinical usefulness of measuring red blood cell distribution width in patients with systemic sclerosis. Rheumatology 2014; 53:1439 1445.

9. Coskun B, Öksüz M, Ermurat S et al. Neutrophil lymphocyte ratio can be a valuable marker in defining disease activity in patients who have started antitumor necrosis factor (TNF) drugs for ankylosing spondylitis. Eur J Rheumatol 2014; 1: 01-5.

10. ARA. Subcommittee for Scleroderma Criteria of the American Rheumatism Association Diagnostic and Therapeutic Criteria Committee. Preliminary criteria for the classification of systemic sclerosis (scleroderma). Arthritis Rheum 1980;23:581-90.

11. Clements J, Lachenbruch A, Seibold R et al. Skin thickness score in systemic sclerosis: an assessment of interobeserver variability in 3 independent studies. J Rheumatol 1993; 20:1892-1896.

12. Czirjak L, Nagy Z, Aringer $M$ et al. The EUSTAR model for teaching and implementing the modified Rodnan skin score in systemic sclerosis. Ann Rheum Dis 2007; 66: 966- 9.

13. Makino $\mathrm{T}$ and Jinnin $\mathrm{M}$. Genetic and epigenetic abnormalities in systemic sclerosis. The Journal of Dermatology 2016;43(1):10-18.

14. Imtiaz F, Shafiue K, Mirza $S$ et al. Neutrophil lymphocyte ratio as a measure of systemic inflammation in prevalent chronic diseases in Asian population. Int Arch Med 2012; 5: 2

15. Guthrie J, Charles A, Roxburgh $\mathrm{S}$ et al. The systemic inflammation-based neutrophillymphocyte ratio: experience in patients with cancer. Crit Rev Oncol Hematol 2013; 88: 218-30.

16. Kaya H, Ertas F, Islamoglu $\mathrm{Y}$ et al. Association between Neutrophil to lymphocyte ratio and severity of coronary artery disease. Clin Appl Thromb Hemost 2014; 20: 50-4.

17. Kalay N, Dogdu O, Koc F et al. Hematologic parameters and angiographic progression of coronary atherosclerosis. Angiology 2012; 63: 213-7.

18. Akpek M, Kaya G, Lam Y et al. Relation of neutrophil/lymphocyte ratio to coronary flow to inhospital major adverse cardiac events in patients with ST-elevated myocardial infarction undergoing primary coronary intervention. Am J Cardiol 2012; 110: 621-7.

19. Uthamalingam S, Patvardhan A, Subramanian S et al. Utility of the neutrophil to lymphocyte ratio in predicting long term outcomes in acute decompensated heart failure. Am J Cardiol 2011; 107: 433-8.

20. Azab B, Zaher M, Weiserbs F et al. Usefulness of neutrophil to lymphocyte ratio in predicting shortand long-term mortality after non-ST-elevation myocardial infarction. Am J Cardiol 2010; 106: 470-6.

21. Biyik M, Ucar R, Solak Y et al. Blood neutrophilto-lymphocyte ratio independently predicts survival in patients with liver cirrhosis. Eur J Gastroenterol Hepatol 2013; 25: 435-41.

22. Forget P, Machiels P, Coulie G et al. Neutrophil: lymphocyte ratio and intraoperative use of ketorolac or diclofenac are prognostic factors in different cohorts of patients undergoing breast, lung, and kidney cancer surgery. Ann Surg Oncol 2013; 20: 650-60.

23. Lee $\mathrm{Y}$, Hong $\mathrm{W}$, Chang $\mathrm{G}$ et al. Clinical significance of preoperative inflammatory parameters in gastric cancer patients. J Gastric Cancer 2013; 13: 111-6.

24. Azab B, Jaglall N, Atallah P et al. Neutrophillymphocyte ratio as a predictor of adverse outcomes of acute pancreatitis. Pancreatology 2011; 11: 445-52.

25. Markar SR, Karthikesalingam A, Falzon A et al. The diagnostic value of neutrophil: lymphocyte ratio in adults with suspected acute appendicitis. Acta Chir Belg 2010; 110: 543-7.

26. Solak Y, Yilmaz I, Sonmez A et al. Neutrophil to lymphocyte ratio independently predicts cardiovascular events in patients with chronic kidney disease. Clin Exp Nephrol 2013; 17: 532-40.

27. Öztürk A, Yesil Y, Kuyumcu E et al. Inverse relationship between neutrophil lymphocyte ratio (NLR) and bone mineral density (BMD) in elderly people. Arch Gerontol Geriatr 2013; 57: 81-5.

28. Ahsen A, Ulu S, Yuksel $\mathrm{S}$ et al. As a New Inflammatory Marker for familial Mediterranean fever: neutrophil-to-lymphocyte Ratio. Inflammation 2013; 36: 1357-62. 